\title{
FLUJO DE ENERGÍA REFLEJADO Y TRANSMITIDO EN CRISTALES FOTÓNICOS UNIDIMENSIONALES
}

\section{REFLECTED ENERGY FLOW AND TRANSMITTED IN ONE-DIMENSIONAL PHOTONIC CRYSTALS}

Francis Segovia Chaves ${ }^{1,2}$

\section{RESUMEN}

Los cristales fotónicos son sistemas cuya principal característica es la periodicidad en el espacio de la función dieléctrica, ellos tienen un principio de funcionalidad simple (la periodicidad), surgiendo así importantes y atractivos efectos en el flujo de la radiación electromagnética. En el presente trabajo se describe el método de transferencia matricial para estudiar la incidencia de una onda electromagnética sobre un cristal fotónico unidimensional, no magnético, binario y sin perdidas. Numéricamente se calcula la dependencia de la reflectancia y transmitancia en función de la longitud de onda del campo electromagnético incidente. Se logra evidenciar la existencia de una región de frecuencias prohibidas en la cual no pueden propagarse los campos a través de la estructura, esto corresponde a una reflectancia máxima. Además se encuentra una dependencia de la región prohibida con la clase de medios que conforman el cristal fotónico, para contrastes mayores del índice de refracción el rango de longitudes de reflectancia es mayor.

Palabras clave: dieléctricos, matriz transferencia, cristal fotónico.

1 Profesor Asistente. Universidad Surcolombiana- Facultad de Ciencias Exactas y Naturales-Programa de Física- Neiva-Huila. 2 Autor corresponsal: francis.segoviac@gmail.com 


\begin{abstract}
Photonic crystals are systems whose main feature is the frequency in the dielectric function space, they have a principle of simple functionality (frequency), emerging and important and interesting effects in the flow of electromagnetic radiation. In this paper the transfer matrix method is described for study the incidence of an electromagnetic wave on a photonic crystal dimensional, nonmagnetic binary without loss. Numerically calculated dependence of reflectance and transmittance versus wavelength of the electromagnetic field. It is achieved to demonstrate the existence of a forbidden frequency region in which the fields cannot be propagated, this corresponds to a maximum reflectance. In addition there is a dependence of the forbidden region with the kind of media that make up the photonic crystal, for contrast over refractive index of the reflectance wavelength range is greater.
\end{abstract}

Keywords: dielectric, transfer matrix, photonic crystal.

\title{
INTRODUCCIÓN
}

El estudio de la propagación de ondas en medios periódicos tiene una larga historia, en los trabajos pioneros de Lord Rayleigh y en el libro de León Brillouin, se interesan por estudiar la propagación en un medio con obstáculos (Rayleigh, 1892; Brillouin, 1953). En 1972 Vladimir P. Bykov propuso la idea de que la emisión espontánea que un cierto átomo produce podría ser inhibida colocándolo en una red periódica en la escala de la longitud de onda de la radiación a emitir. La pérdida de energía por radiación sería imposible si el átomo emite en la región del gap (Bykov, 1972). Estos resultados no tuvieron eco en la comunidad científica sobre la idea de la inhibición de la emisión espontánea. El verdadero interés acerca de la inhibición de la emisión espontánea aparece en 1987, año en que fueron publicados dos trabajos de manera independiente, los cuales marcan el nacimiento de lo que hoy en día se conoce como cristales fotónicos. El primer trabajo hace referencia al investigador Eli Yablanovitch quien trabajaba para la compañía Bell Comunication Research, interesado en obtener materiales en la fabricación de láseres más eficientes para evitar las pérdidas del dispositivo en forma de emisión espontánea que tiene lugar en la producción de emisión estimulada que caracteriza a los láseres (Yablanovitch, 1987). De otro lado, la propuesta de Sajeev Jhon era la utilización de materiales para los cuales en un cierto rango de frecuencias prohibidas, era posible obtener una localización de la luz, al igual que los electrones que quedan confinados en sistemas desordenados (Sajeev, 1991).

Los cristales fotónicos son una nueva especie de semiconductores de luz, en ellos la luz siempre encuentra alguna dirección por la que pueden propagarse a través del cristal y se hace referencia al gap fotónico, como el rango de frecuencias prohibidas donde no existe propagación de la luz. Este tipo de estructuras se caracterizan por presentar modulación periódica en el espacio de su constante dieléctrica.

En 1991 se presenta el primer material con un gap fotónico a partir de una variación de la estructura diamante y que hoy en día se conoce como Yablanovita (Joannopoulos, 1995). En esta estructura se 
utiliza un material con un índice de refracción de 3.6 en la cual se realizan unas perforaciones en disposición triangular a $35.26^{\circ}$ con respecto a la normal y separados a $120^{\circ}$ con respecto al eje azimutal. Debido a que la periodicidad del sistema es de alrededor de la decena de $\mathrm{mm}$, este presenta un gap fotónico en el rango de las microondas.

Es así como los estados permitidos y prohibidos que en cristales atómicos son producidos por medios de fenómenos de interferencia constructiva y destructiva de la función de onda electrónica (Chuang, 1995), en los cristales fotónicos surgen como fenómenos de interferencia de las ondas electromagnéticas dispersadas en el cristal (Kavokin, 2007). Después de 1987 ha existido una gran cantidad de artículos científicos relacionados con cristales fotónicos. En 1996 Thomas Krauss, demostró que era posible obtener una estructura bidimensional en el rango cercano a frecuencias ópticas (Krauss, 1996), de igual manera las investigaciones han estado encaminadas en lograr obtener un cristal fotónico en el rango visibles (Chan, 1998; Berger, 1997; Wijnhoven, 1998) y en la fabricación en el rango de las microondas y en el infrarrojo cercano.

Un elemento de interés para el diseño y posterior utilización de los cristales fotónicos es el conocimiento de la estructura de bandas, es decir, cuales modos pueden ser propagados (modos permitidos) al interior del cristal fotónico. Existen métodos numéricos que permiten obtener información de la estructura de bandas en un cristal fotónico, entre los que se destaca el métodos como la expansión de ondas planas, el método de diferencias finitas en el dominio del tiempo, el método de la matriz de dispersión entre otros (Vasco, 2010; Archuleta, 2007; Guida, 2003). El conocer la estructura de bandas permite conocer las regiones prohibidas de frecuencias en las que ningún modo cuya energía se encuentre en esta región podría propagarse, teniendo como consecuencia que un haz en este rango de energía sea totalmente reflejado por el cristal fotónico (Ozbay, 2004; Notomi, 2000; Yablanovitch, 1993). Valiéndose de lo anterior, hoy en día se diseñan cristales fotónicos con fuentes o medios activos (puntos cuánticos, pozos cuánticos, etc.) que se insertan en el momento de la construcción del cristal, cuyo objetivo es emitir luz en una región para que ésta, al tratar de propagarse, sea reflejada en su totalidad y quede confinada entorno al defecto (Valentim, 2013; Soukoulis, 2002).

En el presente trabajo se estudia el flujo de energía reflejado y transmitido a través de un cristal fotónico unidimensional finito. Para ello haremos uso del método de la matriz transferencia, con el cual podemos determinar los rangos de longitudes de onda del campo electromagnético incidente para que exista o no propagación a lo largo de la estructura. Este trabajo se encuentra dividido principalmente en dos partes: En la sección de materiales y métodos se realiza el planteamiento del problema y se describe el método de la matriz de transferencia, mediante el cual se calcula la reflectancia y transmitancia. Por último se presentan los resultados numéricos obtenidos.

\section{MATERIALES Y MÉTODOS}

Interesa estudiar el flujo de energía transmitido (transmitancia) y reflejado (reflectancia), en el caso de la incidencia de una onda electromagnética plana monocromática sobre un cristal fotónico unidimensional finito, cuya dirección de crecimiento de la estructura se encuentra a lo largo del eje Z. En la Fig. 1 se ilustra el problema a estudiar, el medio de incidencia se caracteriza por un índice de refracción $n_{i}$ y el medio de salida por un índice de refracción $n_{s}$. Se considera que el cristal fotónico está formado por $N$ bicapas de medios dieléctricos alternados no dispersivos y no magnéticos, con índices de refracción $n_{1}$ y $n_{2}$. El espesor de cada uno de los medios que conforman el cristal fotónico es $l_{1}$ y $l_{2}$. 
entrada

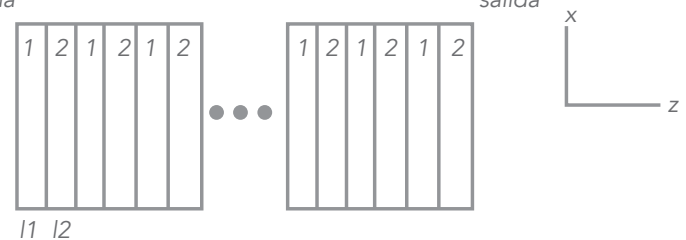

Figura 1. Cristal fotónico unidimensional formado por medios dieléctricos diferentes 1 y 2, caracterizados por índices de refracción $n_{1}$ y $n_{2}$. Los espesores de cada medio viene determinados por $l_{1}$ y $l_{2}$.

A continuación se hace una descripción del método de la matriz de transferencia, fundamentado en las condiciones de frontera que se imponen en cada una de las fronteras de división de los medios que conforman al sistema.

\section{Método de la matriz transferencia}

Las ecuaciones fundamentales de la electrodinámica vienen determinadas por las ecuaciones de Maxwell, en el sistema racionalizado MKS se escriben como (Hecht, 2002):

$$
\begin{gathered}
\vec{\nabla} x \vec{E}=-\frac{\partial \vec{B}}{\partial t}(1) \quad \vec{\nabla} x \vec{H}=\vec{J}+\frac{\partial \vec{D}}{\partial t}(2) \\
\vec{\nabla} \cdot \vec{D}=\rho_{(3)} \quad \vec{\nabla} \cdot \vec{B}=0(4)
\end{gathered}
$$

Donde los vectores de campo vienen determinados por $\vec{E}$ que representa el vector de campo eléctrico, $\vec{D}$ el vector de desplazamiento eléctrico, $\vec{B}$ y $\vec{H}$ representan los vectores de inducción y de campo magnético, respectivamente. Las fuentes de los campos están determinados por la densidad volumétrica de carga eléctrica $\rho$ y el vector de densidad de corriente eléctrica $\vec{J}$. La ecuación (1) hace referencia a la Ley de Faraday en forma diferencial, la cual implica que variaciones temporales del campo magnético son fuentes de campo eléctrico. La ecuación (2), es la ley de Ampere Maxwell, en donde el campo magnético es originado por las densidades de corriente eléctrica y de desplazamiento eléctrico. La ecuación (3) es la Ley de Gauss en forma diferencial, densidades volumétricas de carga eléctrica son fuentes o sumideros del vector de campo eléctrico. Y por último, la no existencia de monopolos magnéticos viene determinada por la ecuación (4). Las ecuaciones de Maxwell se complementan con las ecuaciones constitutivas o relaciones materiales, para medios lineales, homogéneos e isótropos viene dadas por:

$$
\vec{D}=\epsilon \vec{E}(5) \quad \vec{B}=\mu \vec{H}(6)
$$

En la ecuación (5) $\epsilon$ y en la ecuación (6) $\mu$ representan respectivamente los parámetros que caracterizan a un medio material, la permitividad dieléctrica y permeabilidad magnética.

En lo que sigue consideramos que las fuentes de los campos son nulas, $\rho=0$ y $\vec{J}=0$. Los campos electromagnéticos que existen en medios en ausencia de fuentes se denominan ondas electromagnéticas, cuya ecuación de onda para los campos electromagnéticos se puede expresar de la siguiente forma

$$
\left(\nabla^{2}-\epsilon \mu \frac{\partial^{2}}{\partial t^{2}}\right)\left(\begin{array}{l}
\vec{E}(\vec{r}, t) \\
\vec{H}(\vec{r}, t)
\end{array}\right)=0
$$

Las soluciones de interés para las ecuaciones de ondas de los campos electromagnéticos representadas por las ecuaciones (7), son las ondas planas monocromáticas, que se expresan así

$$
\left(\begin{array}{l}
\vec{E}(\vec{r}, t) \\
\vec{H}(\vec{r}, t)
\end{array}\right)=\left(\begin{array}{l}
\vec{E}_{0} \\
\vec{H}_{0}
\end{array}\right) e^{i(\vec{k} \cdot \vec{r}-w t)}
$$

donde $\vec{k}$ representa el vector de onda y w es la frecuencia angular de los campos electromagnéticos. Las amplitudes constantes de los campos electromagnéticos en la ecuación (8) se representan por 
$\vec{E}_{0}$ y $\vec{H}_{0}$, que en general son complejos. Para el caso descrito en la Fig. 1, la onda electromagnética incide sobre la interface de separación haciendo un ángulo $\theta_{i}$ con respecto a la normal. Por lo tanto, es posible descomponer la onda con respecto al plano de incidencia $X Z$ en una componente perpendicular (TE transversal eléctrica) y en una componente paralela (TM transversal magnética).

El vector de onda incidente tiene componentes en $X$ y $Z, \vec{k}_{i}=\left[k_{i x}, 0, k_{i z}\right]$, es decir

$$
\left.\vec{k}_{i}=\frac{w}{c}\left[n_{i} \sin \left(\theta_{i}\right), 0, n_{i} \cos \left(\theta_{i}\right)\right)\right]_{(9)}
$$

En la ecuación (9), c es la velocidad de la luz en el vacío. Tenemos una frontera de separación de dos dieléctricos diferentes de índices de refracción $n_{1}$ y $n_{2}$. Como se supone que los medios son homogéneos en $\mathrm{X}$, los índices de refracción no varían en esa dirección $n(z)$, el módulo del campo eléctrico es:

$$
E(x, z, t)=E(z) e^{i\left(k_{x} x-w t\right)}(10)
$$

la ecuación (10) es válida tanto si con respecto al plano de incidencia, el campo eléctrico es perpendicular o si está contenido en él. A medida que la onda electromagnética avanza a lo largo de la estructura, experimenta múltiples reflexiones en cada una de las interfaces. En este caso $E(z)$, está constituido por la onda que viaja a la derecha $(+Z)$ y otra a la izquierda $(-Z)$ :

$$
\begin{gathered}
E(z)=E_{d}(z) e^{i k_{j z} z}+E_{i}(z) e^{-i k_{j z} z} \\
E(z)=A(z)+B(z)
\end{gathered}
$$

En la ecuación (11), el subíndice j representa el medio dieléctrico, $j=1,2$. La letra $d$ hace referencia a la onda que viaja a la derecha $+Z$ y por $i$ la onda que viaja a la izquierda, $-Z$. En la ecuación (12), se representa la onda que viaja hacia la derecha por $A(z)$ y la que viaja a la izquierda por $B(z)$. En cada uno de los medios dieléctricos los módulos de los campos eléctricos se escriben,

$$
\begin{aligned}
& \text { Medio 1: } E_{1 y}(z)=A_{1}(z)+B_{1}(z)(13) \\
& \text { Medio 2: } E_{2 y}(z)=A_{2}(z)+B_{2}(z)(14)
\end{aligned}
$$

En las ecuaciones (13) y (14), hemos considerado una polarización TE, para el vector de campo eléctrico según el sistema descrito en la Fig. 1. A partir de la ecuación (2), se obtiene el vector de campo magnético en cada medio,

$$
\begin{aligned}
& H_{1 x}(z)=\frac{k_{1} \cos \left(\theta_{1}\right)}{w}\left(A_{1}-B_{1}\right) \\
& H_{2 x}(z)=\frac{k_{2} \cos \left(\theta_{2}\right)}{w}\left(A_{2}-B_{2}\right)
\end{aligned}
$$

En la ecuación (15), $k_{1}$ es el módulo del vector de onda en el medio 1 , viene dado por $k_{1}=\frac{w}{c} n_{1}$. De manera análoga se define en la ecuación (16), el modulo del vector de onda en el segundo medio, $k_{2}={ }_{c}{ }_{c} n_{2}$. Ahora se debe imponer las condiciones de continuidad de la componente tangencial del campo eléctrico en $Y$ y el campo magnético en $X$, para determinar los valores de los parámetros A y B. De lo anterior, se demuestra,

$$
D_{1}\left(\begin{array}{l}
A_{1} \\
B_{1}
\end{array}\right)=D_{2}\left(\begin{array}{l}
A_{2} \\
B_{2}
\end{array}\right)
$$

en la ecuación (17), se define la matriz dinámica para TE en un medio $j(j=1,2)$ como

$$
D_{j}=\left(\begin{array}{cc}
1 & 1 \\
n_{j} \cos \theta_{j} & -n_{j} \cos \theta_{j}
\end{array}\right)_{(18)}
$$


De manera similar a la ecuación (18), la matriz dinámica para TM está dada por

$$
D_{j}=\left(\begin{array}{cc}
\cos \theta_{j} & \cos \theta_{j} \\
n_{j} & -n_{j}
\end{array}\right)
$$

Debe tenerse en cuenta que la onda atraviesa fronteras de división de diferentes medios dieléctricos, se considera que $D_{12}=D_{1}^{-1} D_{2}$ es la matriz de transmisión entre el medio 2 y el medio 1. Además las ondas experimentan un cambio de fase a lo largo de la dirección $Z$, dada por:

$$
\varphi_{j}=k_{j z} l_{j}
$$

La ecuación (20), es positiva para ondas que se propagan a la derecha y negativa si se propagan a la izquierda y $l_{j}$ representa el espesor de los medios dieléctricos que conforman el cristal fotónico. Este cambio de fase se puede representar por una matriz de propagación así:

$$
P_{j}=\left(\begin{array}{cc}
\exp \left(i \varphi_{j}\right) & 0 \\
0 & \exp \left(-i \varphi_{j}\right)
\end{array}\right)
$$

El mecanismo de transmisión y propagación se lleva a cabo a lo largo de toda la estructura fotónica. En general la matriz que característica al sistema de la Fig. 1, se escribe

$$
M=D_{i}^{-1}\left[D_{1} P_{1} D_{i}^{-1} D_{2} P_{2} D_{2}^{-1}\right]^{N} D_{S}
$$

En la ecuación (22), $N$ representa el periodo de las bicapas del cristal fotónico unidimensional. Una vez obtenida la matriz característica, si la onda incide del medio de entrada i y si existe un medio de salida $s$, la transmitancia $T$ y reflectancia $R$ vienen dadas por las siguientes relaciones:

$$
T=\frac{n_{s} \cos \theta_{s}}{n_{i} \cos \theta_{i}}\left|\frac{1}{M_{11}}\right|^{2}, \quad R=\left|\frac{M_{21}}{M_{11}}\right|^{2}
$$

En la ecuación (23), los elementos matriciales $M_{11}$ y $M_{21}$ se encuentran determinados por la matriz característica (22). A continuación presentamos los resultados numéricos obtenidos mediante el método de la matriz transferencia descrito anteriormente, las simulaciones fueron obtenidas por el paquete matemático Mathematica 8.0 (Wolfram, 2003).

\section{RESULTADOS Y DISCUSIÓN}

Teniendo en cuenta el sistema descrito por la Fig. 1, consideramos que el medio de entrada es aire y el de salida es vidrio, los índices de refracción son: $n_{i}=1.0, n_{s}=1.52$. Los índices de refracción para los medios que conforman el cristal fotónicos son: el medio 1 es Cryolita con $n_{1}=1.34$ y el medio 2 es Silicio con $n_{2}=3.4$. Los espesores de cada medio que conforman el cristal fotónico vienen dados por $l_{1}=150 \mathrm{~nm}$ y $l_{2}=100 \mathrm{~nm}$. Teniendo en cuenta la ecuación (23) es posible determinar la reflectancia y transmitancia. En la Fig. 2, se presenta los resultados obtenidos para las curvas de reflectancia y transmitancia en función de la longitud de onda del campo incidente TE con un ángulo de incidencia normal. Se observa que existe un rango de longitudes donde la energía de la onda electromagnética incidente no logra propagarse a través del cristal fotónico, esto corresponde a una región donde la reflectancia es máxima $(R=1)$ y la transmitancia nula $(T=0)$, es decir, en los rangos de longitudes de onda comprendidos aproximadamente entre $550 \mathrm{~nm}$ y $730 \mathrm{~nm}$, y entre $1100 \mathrm{~nm}$ y $1600 \mathrm{~nm}$, se encuentran las regiones de las bandas prohibidas (gap). Por fuera de los intervalos de longitudes de onda anteriormente señalados, existe una transmisión de la energía del campo a lo largo de la estructura. Los resultados concuerdan con los reportados en el trabajo de Aly, (2012).

Además debemos tener en cuenta que en un rango de frecuencias especifico, el ancho de banda prohibido (gap) depende del contrasté de los índices 


\section{UNIVERSIDAD MILITAR NUEVA GRANADA}

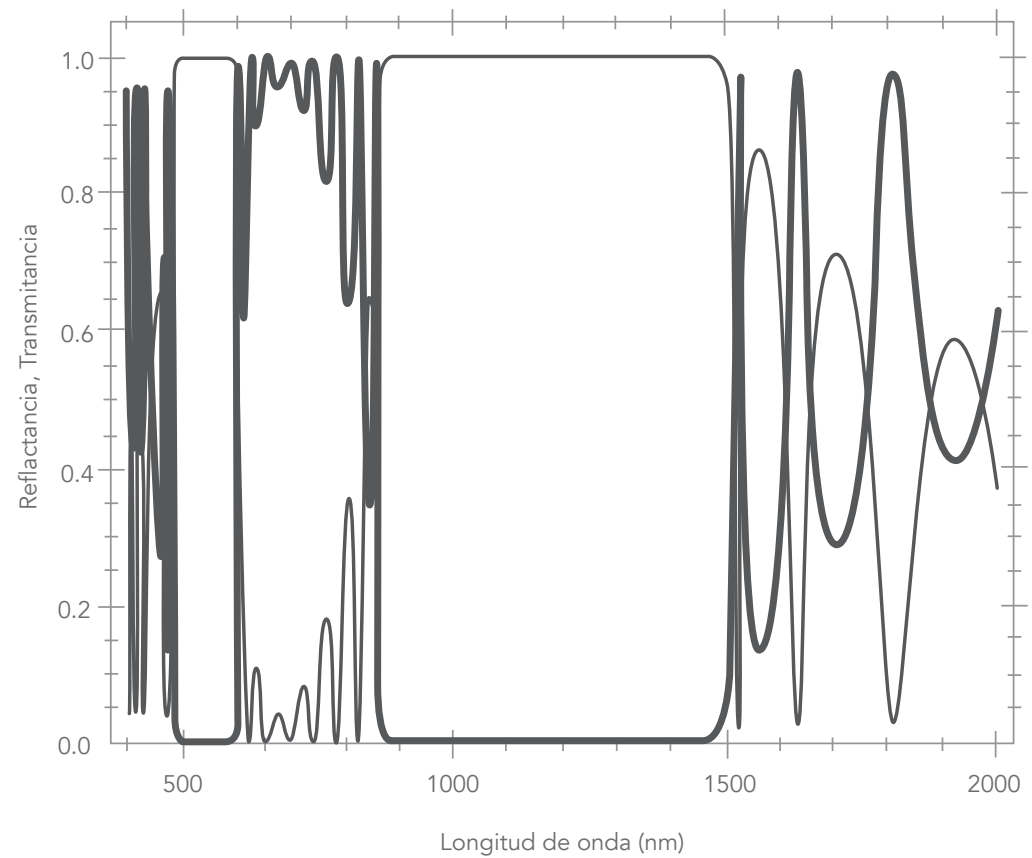

Figura 2. Curvas de reflectancia (línea delgada) y transmitancia (línea gruesa), para una onda electromagnética TE con un ángulo de incidencia $\theta=0^{\circ}$. Se escoge un periodo de $N=10$.

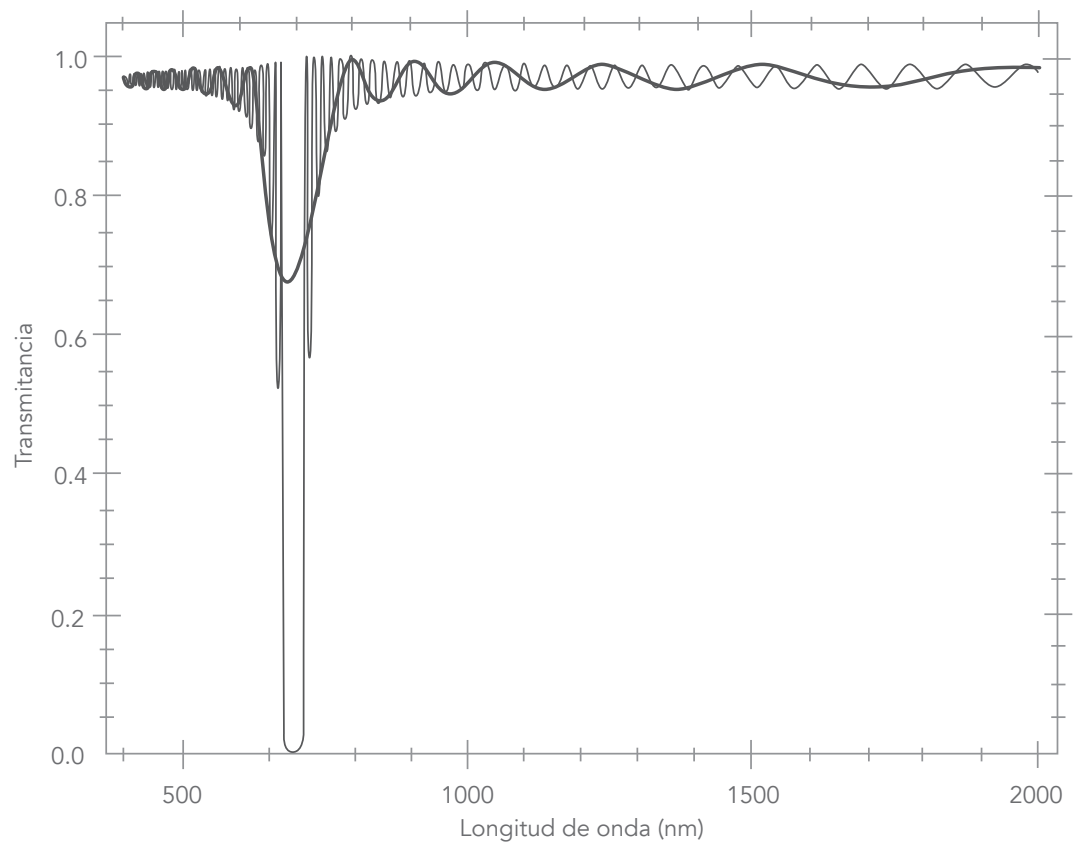

Figura 3. Curvas de transmitancia para un cristal fotónico con periodos de $N=10$ (línea gruesa) y para $N=50$ (línea delgada). La onda electromagnética TE incide normalmente, $\theta=45^{\circ}$. 
de refracción de los dos materiales constituyentes del cristal fotónico. Lo anterior es visible en la Fig. 3 , en ella se presentan las curvas de transmitancia en función de la longitud de onda. Los medios de entrada, salida y el medio 1 son los mismos que se fijaron para obtener la Fig. 2. Sin embargo, se sustituye el medio 2 por dióxido de Cryolita/Silicio con un índice de refracción $n_{2}=1.46$.

Se observa que el número de picos de resonancia de transmisión para el cristal fotónico para un periodo de 50 bicapas es mayor que el número de picos de transmisión con un periodo de 10 bicapas. Notamos que los picos de resonancia de transmisión son directamente proporcionales al número de periodos que conforman al cristal fotónico, los resultados coinciden a los presentados por Aly, (2012).

A medida que el ángulo de incidencia se incrementa, afecta los resultados de transmitancia para el cristal fotónico. En la Fig. 4, se representa las curvas de transmitancia con los parámetros utilizados para obtener la Fig. 3,en ella se evidencia que al aumentar el ángulo de incidencia de la onda electromagnética a $\theta=45^{\circ}$, el gap de frecuencia prohibido se incrementa considerablemente con respecto al resultado reportado en la Fig. 3.

Una aplicación muy importante de los cristales fotónicos es su utilización como reflectores de Bragg, en ellos se impone la condición que los espesores del dispositivo cumplen con la condición de cuarto de onda $\left(n_{1} l_{1}=n_{2} l_{2}=\frac{\lambda_{0}}{4}\right)$, siendo $\lambda_{0}$ la longitud de onda de diseño. En la Fig. 5, se presenta la curva de transmitancia en función de la longitud de onda.

Los parámetros escogidos en la Fig. 5 son: el medio de entrada es aire y el de salida es vidrio, los índices de refracción correspondientes son $n_{i}=1.0$ y $n_{s}=1.52$, el medio 1 se caracteriza por un índice $n_{1}=3.3$ y el medio 2 es MgF2 con un índice de refracción $n_{2}=1.3855$. Los espesores de cada medio que conforman el cristal fotónico



Figura 4. Curvas de transmitancia para un cristal fotónico con periodos de $N=10$ (línea gruesa) y para $N=50$ (línea delgada). La onda electromagnética TE incide con un ángulo $\theta=45^{\circ}$. 
del dispositivo son $l_{1}=22.73 \mathrm{~nm}$ y $l_{2}=54.13 \mathrm{~nm}$, los cuales satisfacen la condición de cuarto de onda para una longitud de onda de diseño de $300 \mathrm{~nm}$. Como se muestra en la Fig. 5, existe una amplia banda de frecuencias prohibidas dentro de la región UV (ultravioleta), esto es debido al alto contraste de índices de refracción del MgF2 y el material de menos perdida. Los resultados obtenidos concuerdan con los reportados en presentados en el trabajo de Arafa, (2012).

\section{CONCLUSIONES}

La propiedad inusual de los cristales fotónicos de permitir y prohibir la propagación de ondas electromagnéticas para ciertos rangos de longitudes de onda y frecuencias, hacen que este tipo de estructuras sean ampliamente usadas como guías de onda en telecomunicaciones. En el presente trabajo se describió analíticamente el método de la matriz de transferencia que caracteriza a un cristal fotónico unidimensional junto con las relaciones que definen la reflectancia y transmitancia. Los resultados que se obtuvieron coincidieron con los reportados en la literatura científica. Se evidenció la importancia de los medios que conforman el cristal, ya que para contrastes mayores del índice de refracción el rango de longitudes de reflectancia es mayor y por ello se evidencia una región mayor del gap de frecuencias prohibidas. De igual manera se mostró que estructuras fotónicas con un apilamiento mayor de periodos, los picos de transmitancia aumenta proporcionalmente.

\section{AGRADECIMIENTOS}

A la Universidad Surcolombiana por su apoyo financiero mediante el proyecto de investigación: Masas y mezclas de los neutrinos a partir de operadores autoadjuntos positivos asociados a espacios de Hilbert.



Figura 5. Curvas de reflectancia (línea delgada) y transmitancia (línea gruesa), para una onda electromagnética TE con un ángulo de incidencia $\theta=0^{\circ}$ y con un periodo de $N=10$. 


\section{REFERENCIAS}

1. Aly A., Ismaeel M., Rahman E. 2012. Comparative study of the one dimensional dielectric and metallic photonic crystals. Optics and Photonics Journal, 2: 105-112.

2. Arafa A., Elsayed A. 2012. Defect mode properties in a one dimensional photonic crystal. Physica B, 407: 120-125.

3. Archuleta A., Manzanares M., Manzanares J. 2007. Una descripción del método de ondas planas para el cálculo de bandas fotónicas. Revista Boliviana de Física, 13: 79-85.

4. Berger V., Gauthier L., Costard, E. 1997. Photonic band gaps and holography. Journal of Applied Physics, 82: pp. 60-64.

5. Brillouin L. 1953. Wave propagation in periodic structures. Dover Publications, New York, 4p.

6. Bykov V. 1972. Frecuency scanning in a lasser with an active negative feedback. Dockl. Akad. Nauka SSSR, 206: 1078-1079.

7. Chan C., Datta H., Soukoulis C. 1998. A7 structure: A family of photonic crystals. Physics Review B, 50: 802-804.

8. Chuang S., 1995. Physics of optoelectronics devices. John Wiley and Sons, New York, 223p.

9. Guida G., Lustrac A., Priou A. 2003. An introduction to photonic band gap (PBG) materials. Progress in Electromagnetic Research, 41: 1-20.

10. Hecht E., 2002. Optics. Addison Wesley, New York, 100p

11. Joannopoulos J., Meade R., Winn J. 1995. Photonic Crystals: Molding the Flow of Light. Princenton University Press, New York, 189p.

12. Kavokin A., Baumberg J., Malpuech G., Laussy F. 2007. Microcavities. Oxford Science Publications, New York, 134p.

13. Krauss T., DeLaRue R., Brand S. 1996. Two dimensional photonic bandgap structures operating at near infrared wavelengths. Nature, 383: 699-702.

14. Notomi M. 2000. Theory of light propagation in strongly modulated photonic crystals: Refractionlike behavior in the vicinity of the photonic band gap. Physical Review B, 62: 10696-1075.

15. Ozbay E., Bulu I., Aydin K., Caglayan H. 2004. Physics and applications of photonic crystal. Photonics and Nanostructures-Fundamentals and Applications, 2: 87-95.

16. Rayleigh L. 1892. On the influence of obstacles arranged in rectangular order upon the properties of a medium. Phylos. Mag., 34: 481-482.

17. Sajeev J. 1991. Localization of light. Physics Today: $32-40$.

18. Soukoulis C. 2002. The history and a review of the modeling and fabrication of photonic crystals. Nanotechnology, 13: 420-423.

19. Vasco J., Vinck H. 2010. Método de diferencias finitas en el dominio de las frecuencias para cristales fotónicos 1D y 2D. Revista Tecnológicas, 24: 125-141.

20. Valentim P. 2013. Um estudo sobre cristais fotónicos 1D e 2D. Tesis Doctorado, Programa de Fisica, Universidad Federal De Minas Gerais, Sao Paulo, Brasil, 107p.

21. Wijnhoven J., Vos W. 1998. Preparation of photonic crystals made of air spheres in titania. Science, 281: 802-804.

22. Wolfram, S., 2003. Mathematica Book. Published by Wolfram Media, New York, 569p.

23. Yablanovitch E. 1987. Inhibited spontaneous emission in solid state physics and electronics. Physics Review Letters, 58: 2059-2062.

24. Yablanovitch E. 1993. Photonic band-gap crystals. Journal Physics Condensate Matter, 5: 2443-2460. 